\title{
On the k-Fibonacci words
}

\author{
José L. RAMÍREZ \\ Instituto de Matemáticas y sus \\ Aplicaciones \\ Universidad Sergio Arboleda, Colombia \\ email: \\ Gustavo N. RUBIANO \\ Departamento de Matemáticas \\ Universidad Nacional de Colombia, \\ Colombia \\ email: gnrubianoo@unal.edu.co \\ josel.ramirez@ima.usergioarboleda.edu.co
}

\begin{abstract}
In this paper we define the k-Fibonacci words in analogy with the definition of the k-Fibonacci numbers. We study their properties and we associate to this family of words a family of curves with interesting patterns.
\end{abstract}

\section{Introduction}

Fibonacci numbers and their generalizations have many interesting properties and applications to almost every fields of science and arts (e.g. see [13]). The Fibonacci numbers $F_{n}$ are the terms of the sequence $0,1,1,2,3,5, \ldots$ wherein each term is the sum of the two previous terms, beginning with the values $\mathrm{F}_{0}=0$, and $\mathrm{F}_{1}=1$.

Besides the usual Fibonacci numbers many kinds of generalizations of these numbers have been presented in the literature. In particular, a generalization is the k-Fibonacci numbers [11].

For any positive real number $k$, the $k$-Fibonacci sequence, say $\left\{F_{k, n}\right\}_{n \in \mathbb{N}}$ is defined recurrently by

$$
F_{k, 0}=0, F_{k, 1}=1 \text { and } F_{k, n+1}=k F_{k, n}+F_{k, n-1}, n \geqslant 1 .
$$

Computing Classification System 1998: I.3.7, G.2.0

Mathematics Subject Classification 2010: 11B39, 05A05, 68R15

Key words and phrases: Fibonacci word, k-Fibonacci numbers, k-Fibonacci words, kFibonacci curves 
In [11], k-Fibonacci numbers were found by studying the recursive application of two geometrical transformations used in the four-triangle longest-edge (4TLE) partition. These numbers have been studied in several papers, see $[5,10,11,12,18,19,23]$.

The characteristic equation associated to the recurrence relation (1) is $x^{2}=$ $k x+1$. The roots of this equation are

$$
r_{k, 1}=\frac{k+\sqrt{k^{2}+4}}{2} \text {, and } r_{k, 2}=\frac{k-\sqrt{k^{2}+4}}{2} \text {. }
$$

Some of the properties that the k-Fibonacci numbers verify are (see $[11,12]$ for the proofs).

- Binet Formula: $F_{k, n}=\frac{r_{k, 1}^{n}-r_{k, 2}^{n}}{r_{k, 1}-r_{k, 2}}$.

- Combinatorial Formula: $F_{k, n}=\sum_{i=0}^{\left\lfloor\frac{n-1}{2}\right\rfloor}\left(\begin{array}{c}n-1-i \\ i\end{array}\right) k^{n-1-2 i}$.

- $\lim _{n \rightarrow \infty} \frac{F_{k, n}}{F_{k, n-1}}=r_{k, 1}$.

On the other hand, there is a word-combinatorial interpretation of the Fibonacci sequence. Fibonacci words are words over $\{0,1\}$ defined recursively as follows:

$$
f_{0}=1, \quad f_{1}=0, \quad f_{n}=f_{n-1} f_{n-2}, \quad n \geqslant 2 .
$$

The words $f_{n}$ are referred to as the finite Fibonacci words and it is clear that $\left|f_{n}\right|=F_{n+1}$. The limit

$$
\mathbf{f}=\lim _{n \rightarrow \infty} f_{n}=0100101001001010010100100101 \cdots
$$

is called the Fibonacci word. This word is certainly one of the most studied words in the combinatorics on words, (see, e.g., $[2,6,7,9,15,22]$ ). It is the archetype of a Sturmian word [14]. This word can be associated with a curve, which has fractal properties obtained from combinatorial properties of f $[3,16,21]$.

In this paper we introduce a family of words $\mathbf{f}_{\mathrm{k}}$ that generalize the Fibonacci word. Specifically, the k-Fibonacci words are words over $\{0,1\}$ defined inductively as follows

$$
f_{k, 0}=0, \quad f_{k, 1}=0^{k-1} 1, \quad f_{k, n}=f_{k, n-1}^{k} f_{k, n-2},
$$


for all $n \geq 2$ and $k \geq 1$. Then it is clear that $\left|f_{k, n}\right|=F_{k, n+1}$. The infinite word

$$
\mathbf{f}_{k}:=\lim _{n \rightarrow \infty} f_{k, n}
$$

is called the k-Fibonacci word. In connection with this definition, we investigate some new combinatorial properties and we associate a family of curves with interesting patterns.

\section{Definitions and notation}

The terminology and notations are mainly those of Lothaire [14] and Allouche and Shallit [1].

Let $\Sigma$ be a finite alphabet, whose elements are called symbols. A word over $\Sigma$ is a finite sequence of symbols from $\Sigma$. The set of all words over $\Sigma$, i.e., the free monoid generated by $\Sigma$, is denoted by $\Sigma^{*}$. The identity element $\epsilon$ of $\Sigma^{*}$ is called the empty word and $\Sigma^{+}=\Sigma^{*} \backslash\{\epsilon\}$. For any word $w \in \Sigma^{*},|w|$ denotes its length, i.e., the number of symbols occurring in $w$. The length of $\epsilon$ is taken to be equal to 0 . If $a \in \Sigma$ and $w \in \Sigma^{*}$, then $|w|_{a}$ denotes the number of occurrences of $a$ in $w$.

For two words $u=a_{1} a_{2} \cdots a_{k}$ and $v=b_{1} b_{2} \cdots b_{s}$ in $\Sigma^{*}$ we denote by $u v$ the concatenation of the two words, that is, $u v=a_{1} a_{2} \cdots a_{k} b_{1} b_{2} \cdots b_{s}$. If $v=\epsilon$ then $\mathfrak{u} \epsilon=\epsilon \mathfrak{u}=\mathfrak{u}$, moreover, by $\mathfrak{u}^{\mathfrak{n}}$ we denote the word $\mathfrak{u} u \cdots \mathfrak{u}$ ( $n$ times). A word $v$ is a subword (or factor) of $u$ if there exist $x, y \in \Sigma^{*}$ such that $u=x v y$. If $x=\epsilon(y=\epsilon)$, then $v$ is called prefix (suffix) of $u$.

The reversal of a word $u=a_{1} a_{2} \cdots a_{n}$ is the word $u^{R}=a_{n} \cdots a_{2} a_{1}$ and $\epsilon^{R}=\epsilon$. A word $u$ is a palindrome if $u^{R}=u$.

An infinite word over $\Sigma$ is a map $\mathbf{u}: \mathbb{N} \rightarrow \Sigma$. It is written $\mathbf{u}=a_{1} a_{2} a_{3} \cdots$. The set of all infinite words over $\Sigma$ is denoted by $\Sigma^{\omega}$.

Example 1 The word $\mathbf{p}=\left(p_{n}\right)_{n \geq 1}=0110101000101 \cdots$, where $p_{n}=1$ if $n$ is a prime number and $\mathrm{p}_{\mathrm{n}}=0$ otherwise, is an example of an infinite word. $\mathbf{p}$ is called the characteristic sequence of the prime numbers.

Definition 2 Let $\Sigma$ and $\Delta$ be alphabets. A morphism is a map $\mathrm{h}: \Sigma^{*} \rightarrow \Delta^{*}$ such that $\mathrm{h}(\mathrm{xy})=\mathrm{h}(\mathrm{x}) \mathrm{h}(\mathrm{y})$ for all $\mathrm{x}, \mathrm{y} \in \Sigma^{*}$. It is clear that $\mathrm{h}(\epsilon)=\epsilon$.

There is a special class of words, with many remarkable properties, the socalled Sturmian words. These words admit several equivalent definitions (see, e.g. [1] or [14]). 
Definition 3 Let $\mathbf{w} \in \Sigma^{\omega}$. We define $\mathrm{P}(\mathbf{w}, \mathfrak{n})$, the complexity function of $\mathbf{w}$, to be the map that counts, for all integer $\mathrm{n} \geq 0$, the number of subwords of length $\mathrm{n}$ in $\mathbf{w}$. An infinite word $\mathbf{w}$ is a Sturmian word if $\mathrm{P}(\mathbf{w}, \mathrm{n})=\mathrm{n}+1$ for all integer $\mathrm{n} \geq 0$.

Since for any Sturmian word $\mathrm{P}(\mathbf{w}, 1)=2$, then Sturmian words are over two symbols. The word $\mathbf{p}$, in Example 1, is not a Sturmian word because $\mathrm{P}(\mathbf{p}, 2)=4$.

Given two real numbers $\alpha, \beta \in \mathbb{R}$ with $\alpha$ irrational and $0<\alpha<1,0 \leq \beta<1$, we define the infinite word $\mathbf{w}=w_{1} w_{2} w_{3} \cdots$ as

$$
w_{n}=\lfloor(n+1) \alpha+\beta\rfloor-\lfloor n \alpha+\beta\rfloor .
$$

The numbers $\alpha$ and $\beta$ are the slope and the intercept, respectively. This word is called mechanical. The mechanical words are equivalent to Sturmian words [14]. As special case, when $\beta=0$, we obtain the characteristic words.

Definition 4 Let $\alpha$ be an irrational number with $0<\alpha<1$. For $\mathrm{n} \geq 1$, define

$$
w_{\alpha}(n):=\lfloor(n+1) \alpha\rfloor-\lfloor n \alpha\rfloor,
$$

and

$$
\mathbf{w}(\alpha):=w_{\alpha}(1) w_{\alpha}(2) w_{\alpha}(3) \cdots
$$

then $\mathbf{w}(\alpha)$ is called a characteristic word with slope $\alpha$.

On the other hand, note that every irrational $\alpha \in(0,1)$ has a unique continued fraction expansion

$$
\alpha=\left[0, a_{1}, a_{2}, a_{3}, \ldots\right]=\frac{1}{a_{1}+\frac{1}{a_{2}+\frac{1}{a_{3}+\cdots}}},
$$

where each $a_{i}$ is a positive integer. Let $\alpha=\left[0,1+d_{1}, d_{2}, \ldots\right]$ be an irrational number with $d_{1} \geq 0$ and $d_{n}>0$ for $n>1$. To the directive sequence $\left(d_{1}, d_{2}, \ldots, d_{n}, \ldots\right)$, we associate a sequence $\left(s_{n}\right)_{n \geq-1}$ of words defined by

$$
s_{-1}=1, \quad s_{0}=0, \quad s_{n}=s_{n-1}^{d_{n}} s_{n-2}, \quad(n \geq 1) .
$$


Such a sequence of words is called a standard sequence. This sequence is related to characteristic words in the following way. Observe that, for any $n \geq 0, s_{n}$ is a prefix of $s_{n+1}$, which gives meaning to $\lim _{n \rightarrow \infty} s_{n}$ as an infinite word. In fact, one can prove [14] that each $s_{n}$ is a prefix of $\mathbf{w}(\alpha)$ for all $n \geq 0$ and

$$
\mathbf{w}(\alpha)=\lim _{n \rightarrow \infty} s_{n}
$$

Example 5 The infinite Fibonacci word $\mathbf{f}=0100101001001010 \cdots$ is a Sturmian word [14], exactly $\mathbf{f}=\mathbf{w}\left(\frac{1}{\phi^{2}}\right)$ where $\phi=\frac{1+\sqrt{5}}{2}$ is the golden ratio.

Definition 6 The Fibonacci morphism $\sigma:\{0,1\} \rightarrow\{0,1\}$ is defined by $\sigma(0)=$ 01 and $\sigma(1)=0$.

The Fibonacci word $\mathbf{f}$ satisfies that $\lim _{\mathfrak{n} \rightarrow \infty} \sigma^{\mathfrak{n}}(1)=\mathbf{f}[1]$.

\section{The k-Fibonacci words}

Definition 7 The $\mathrm{n}$ th $\mathrm{k}$-Fibonacci words are words over $\{0,1\}$ defined inductively as follows

$$
f_{k, 0}=0, \quad f_{k, 1}=0^{k-1} 1, \quad f_{k, n}=f_{k, n-1}^{k} f_{k, n-2},
$$

for all $\mathrm{n} \geq 2$ and $\mathrm{k} \geq 1$. The infinite word

$$
\mathbf{f}_{k}:=\lim _{n \rightarrow \infty} f_{k, n}
$$

is called the k-Fibonacci word.

It is clear that $\left|f_{k, n}\right|=F_{k, n+1}$. For $k=1$ we have the word $\overline{\mathbf{f}}=1011010110110 \ldots$, where $\bar{a}$ is a morphism, with $a \in\{0,1\}$, defined by $\overline{0}=1, \overline{1}=0$.

Example 8 The first $\mathrm{k}$-Fibonacci words are

$$
\begin{array}{lll}
\mathbf{f}_{1}=1011010110110 \cdots=\overline{\mathbf{f}}, & \mathbf{f}_{2}=0101001010010 \cdots, & \mathbf{f}_{3}=0010010010001 \cdots, \\
\mathbf{f}_{4}=0001000100010 \cdots, & \mathbf{f}_{5}=0000100001000 \cdots, & \mathbf{f}_{6}=0000010000010 \cdots
\end{array}
$$

Definition 9 The $k$-Fibonacci morphism $\sigma_{k}:\{0,1\} \rightarrow\{0,1\}$ is defined by $\sigma_{k}(0)=0^{k-1} 1$ and $\sigma_{k}(1)=0^{k-1} 10$.

Theorem 10 For all $\mathrm{n} \geq 0, \sigma_{k}^{n}(0)=f_{k, n}$ and $\sigma_{k}^{n+1}(1)=f_{k, n+1} f_{k, n}$. Hence, the $\mathrm{k}$-Fibonacci word $\mathbf{f}_{\mathrm{k}}$ satisfies that $\lim _{\mathrm{n} \rightarrow \infty} \sigma^{\mathfrak{n}}(0)=\mathbf{f}_{\mathrm{k}}$. 
Proof. We prove the two assertions about $\sigma_{k}^{n}$ by induction on $n$. They are clearly true for $n=0,1$. Assume for all $j<n$; we prove them for $n$ :

$$
\begin{aligned}
\sigma_{k}^{n+1}(0) & =\sigma_{k}^{n}\left(0^{k-1} 1\right)=\left(\sigma_{k}^{n}(0)\right)^{k-1} \sigma_{k}^{n}(1)=f_{k, n}^{k-1} f_{k, n} f_{k, n-1}=f_{k, n}^{k} f_{k, n-1}=f_{k, n+1} . \\
\sigma_{k}^{n+2}(1) & =\sigma_{k}^{n+1}\left(0^{k-1} 10\right)=\left(\sigma_{k}^{n+1}(0)\right)^{k-1} \sigma_{k}^{n+1}(1) \sigma_{k}^{n+1}(0)=f_{k, n+1}^{k-1} f_{k, n+1} f_{k, n} f_{k, n+1} \\
& =f_{k, n+1}^{k} f_{k, n} f_{k, n+1}=f_{k, n+2} f_{k, n+1} .
\end{aligned}
$$

\section{Proposition 11}

1. $\left|f_{k, n}\right|_{1}=F_{k, n}$ and $\left|f_{k, n+1}\right|_{0}=F_{k, n+1}+F_{k, n}$ for all $n \geqslant 0$.

2. $\lim _{n \rightarrow \infty} \frac{\left|f_{k, n}\right|}{\left|f_{k, n}\right|_{0}}=\frac{r_{k, 1}^{2}}{1+r_{k, 1}}$.

3. $\lim _{n \rightarrow \infty} \frac{\left|f_{k, n}\right|}{\left|f_{k, n}\right|_{1}}=r_{k, 1}$.

4. $\lim _{n \rightarrow \infty} \frac{\left|f_{k, n}\right|_{0}}{\left|f_{k, n}\right|_{1}}=1+\frac{1}{r_{k, 1}}$.

\section{Proof.}

1. It is clear by induction on $n$.

2. $\lim _{n \rightarrow \infty} \frac{\left|f_{k, n}\right|}{\left|f_{k, n}\right|_{0}}=\lim _{n \rightarrow \infty} \frac{F_{k, n+1}}{F_{k, n}+F_{k, n-1}}=\lim _{n \rightarrow \infty} \frac{\frac{F_{k, n+1}}{F_{k, n}}}{1+\frac{F_{k, n-1}}{F_{k, n}}}=\frac{r_{k, 1}^{2}}{1+r_{k, 1}}$.

3. $\lim _{n \rightarrow \infty} \frac{\left|f_{k, n}\right|}{\left|f_{k, n}\right|_{1}}=\lim _{n \rightarrow \infty} \frac{F_{k, n+1}}{F_{k, n}}=r_{k, 1}$.

4. $\lim _{n \rightarrow \infty} \frac{\left|f_{k, n}\right|_{0}}{\left|f_{k, n}\right|_{1}}=\lim _{n \rightarrow \infty} \frac{F_{k, n}+F_{k, n-1}}{F_{k, n}}=1+\frac{1}{r_{k, 1}}$.

Proposition 12 The k-Fibonacci word and the $\mathrm{n}$ th $\mathrm{k}$-Fibonacci word satisfy that

1. The word 11 is not a subword of the $\mathrm{k}$-Fibonacci word, $\mathrm{k} \geq 2$.

2. Let $\mathrm{ab}$ be the last two symbols of $\mathrm{f}_{\mathrm{k}, \mathrm{n}}$. For $\mathrm{n} \geq 1$, we have $\mathrm{ab}=10$ if $\mathrm{n}$ is even and $\mathrm{ab}=01$ if $\mathrm{n}$ is odd, $\mathrm{k} \geq 2$.

3. The concatenation of two successive k-Fibonacci words is "almost commutative", i.e., $\mathrm{f}_{\mathrm{k}, \mathrm{n}-1} \mathrm{f}_{\mathrm{k}, \mathrm{n}-2}$ and $\mathrm{f}_{\mathrm{k}, \mathrm{n}-2} \mathrm{f}_{\mathrm{k}, \mathrm{n}-1}$ have a common prefix the length $\mathrm{F}_{\mathrm{k}, \mathrm{n}}+\mathrm{F}_{\mathrm{k}, \mathrm{n}-1}-2$ for all $\mathrm{n} \geq 2$. 


\section{Proof.}

1. It suffices to prove that 11 is not a subword of $f_{k, n}$, for all $n \geq 0$. By induction on $n$. For $n=0,1$ it is clear. Assume for all $j<n$; we prove it for $n$. We know that $f_{k, n}=f_{k, n-1}^{k} f_{k, n-2}$ so by the induction hypothesis we have that 11 is not a subword of $f_{k, n-1}$ and $f_{k, n-2}$. Therefore, the only possibility is that 1 is a suffix and prefix of $f_{k, n-1}$ or 1 is a suffix of $f_{k, n-1}$ and a prefix of $f_{k, n-2}$, both there are impossible.

2. By induction on $\mathfrak{n}$. For $\mathfrak{n}=1,2$ it is clear. Assume for all $j<n$; we prove it for $n$. We know that $f_{k, n+1}=f_{k, n}^{k} f_{k, n-1}$, if $n+1$ is even then by the induction hypothesis the last two symbols of $f_{k, n-1}$ are 10 , therefore the last two symbols of $f_{k, n+1}$ are 10. Analogously, if $n+1$ is odd.

3. By induction on $n$. For $n=1,2$ it is clear. Assume for all $j<n$; we prove it for $n$. By definition of $f_{k, n}$, we have

$$
\begin{aligned}
f_{k, n-1} f_{k, n-2} & =f_{k, n-2}^{k} f_{k, n-3} \cdot f_{k, n-3}^{k} f_{k, n-4} \\
& =\left(f_{k, n-3}^{k} f_{k, n-4}\right)^{k} \cdot f_{k, n-3}^{k} f_{k, n-3} f_{k, n-4},
\end{aligned}
$$

and

$$
\begin{aligned}
f_{k, n-2} f_{k, n-1} & =f_{k, n-3}^{k} f_{k, n-4} \cdot f_{k, n-2}^{k} f_{k, n-3} \\
& =f_{k, n-3}^{k} f_{k, n-4} \cdot\left(f_{k, n-3}^{k} f_{k, n-4}\right)^{k} \cdot f_{k, n-3} \\
& =\left(f_{k, n-3}^{k} f_{k, n-4}\right)^{k} f_{k, n-3}^{k} f_{k, n-4} f_{k, n-3} .
\end{aligned}
$$

Hence the words have a common prefix of length $k\left(k F_{k, n-2}+F_{k, n-3}\right)+$ $k F_{n-2}=k\left(F_{k, n-1}+F_{k, n-2}\right)$. By the induction hypothesis $f_{k, n-3} f_{k, n-4}$ and $f_{k, n-4} f_{k, n-3}$ have a common prefix of length $F_{k, n-2}+F_{k, n-3}-2$. Therefore the words have a common prefix of length

$$
k\left(F_{k, n-1}+F_{k, n-2}\right)+F_{k, n-2}+F_{k, n-3}-2=F_{k, n}+F_{k, n-1}-2 .
$$

Definition 13 Let $\Phi:\{0,1\}^{*} \rightarrow\{0,1\}^{*}$ be a map such that $\Phi$ deletes the last two symbols, i.e., $\Phi\left(a_{1} a_{2} \cdots a_{n}\right)=a_{1} a_{2} \cdots a_{n-2}(n \geq 2)$.

Corollary 14 The $\mathrm{n}$ th $\mathrm{k}$-Fibonacci word, satisfy for all $\mathrm{n} \geqslant 2$ that

1. $\Phi\left(f_{k, n-1} f_{k, n-2}\right)=\Phi\left(f_{k, n-2} f_{k, n-1}\right)$.

2. $\Phi\left(f_{k, n-1} f_{k, n-2}\right)=f_{k, n-2} \Phi\left(f_{k, n-1}\right)=f_{k, n-1} \Phi\left(f_{k, n-2}\right)$.

3. If $f_{k, n}=\Phi\left(f_{k, n}\right) a b$, then $\Phi\left(f_{k, n-2}\right) a b \Phi\left(f_{k, n-1}\right)=f_{k, n-1} \Phi\left(f_{k, n-2}\right)$. 


\section{If $f_{k, n}=\Phi\left(f_{k, n}\right) a b$, then $\Phi\left(f_{k, n-2}\right)\left(a b \Phi\left(f_{k, n-1}\right)\right)^{k}=\Phi\left(f_{k, n}\right)$.}

Proof. Parts (a) and (b) follow immediately from Proposition 12-(3) and because of $\left|f_{k, n}\right| \geqslant 2$ for all $n \geqslant 2$. (c) In fact, if $f_{k, n}=\Phi\left(f_{k, n}\right)$ ab then from Proposition 12-(2) we have $f_{k, n-2}=\Phi\left(f_{k, n-2}\right) a b$. Hence $\Phi\left(f_{k, n-2}\right) a b \Phi\left(f_{k, n-1}\right)=$ $f_{k, n-2} \Phi\left(f_{k, n-1}\right)=f_{k, n-1} \Phi\left(f_{k, n-2}\right)$. (d) It is clear from (c) and definition of $f_{k, n}$.

Theorem $15 \Phi\left(f_{k, n}\right)$ is a palindrome for all $\mathrm{n} \geq 1$ and $\mathrm{k} \geq 1$.

Proof. By induction on $n$. If $n=2$ then $\Phi\left(f_{k, 2}\right)=\left(0^{k-1} 1\right)^{k-1} 0^{k-1}$ is a palindrome. Now suppose that the result is true for all $j<n$; we prove it for n.

$$
\begin{aligned}
\left(\Phi\left(f_{k, n}\right)\right)^{R} & =\left(\Phi\left(f_{k, n-1}^{k} f_{k, n-2}\right)\right)^{R}=\left(f_{k, n-1}^{k} \Phi\left(f_{k, n-2}\right)\right)^{R}=\Phi\left(f_{k, n-2}\right)^{R}\left(f_{k, n-1}^{k}\right)^{R} \\
& =\Phi\left(f_{k, n-2}\right)\left(f_{k, n-1}^{R}\right)^{k} .
\end{aligned}
$$

If $n$ is even then $f_{k, n}=\Phi\left(f_{k, n}\right) 10$ and from Corollary 14-(4), we have that

$$
\begin{aligned}
\left(\Phi\left(f_{k, n}\right)\right)^{R} & =\Phi\left(f_{k, n-2}\right)\left(\left(\Phi\left(f_{k, n-1}\right) 01\right)^{R}\right)^{k}=\Phi\left(f_{k, n-2}\right)\left(10\left(\Phi\left(f_{k, n-1}\right)\right)^{R}\right)^{k} \\
& =\Phi\left(f_{k, n-2}\right)\left(10 \Phi\left(f_{k, n-1}\right)\right)^{k}=\Phi\left(f_{k, n}\right) .
\end{aligned}
$$

If $n$ is odd, the proof is analogous.

Corollary 16 1. If $\mathrm{f}_{\mathrm{k}, \mathrm{n}}=\Phi\left(\mathrm{f}_{\mathrm{k}, \mathrm{n}}\right) \mathrm{ab}$ then $\mathrm{ba} \Phi\left(\mathrm{f}_{\mathrm{k}, \mathrm{n}}\right) \mathrm{ab}$ is a palindrome. 2. If $\mathrm{u}$ is a subword of the $\mathrm{k}$-Fibonacci word, then so is its reversal, $\mathrm{u}^{\mathrm{R}}$.

Theorem 17 Let $\alpha=[0, \overline{\mathrm{k}}]$ be an irrational number, with $\mathrm{k}$ a positive integer, then

$$
\mathbf{w}(\alpha)=\mathbf{f}_{\mathrm{k}}
$$

Proof. Let $\alpha=[0, \bar{k}]$ an irrational number, then its associated standard sequence is

$$
s_{-1}=1, \quad s_{0}=0, \quad s_{1}=s_{0}^{k-1} s_{-1}=0^{k-1} 1 \text { and } s_{n}=s_{n-1}^{k} s_{n-2}, n \geq 2 .
$$

Hence $\left\{s_{\mathfrak{n}}\right\}_{\mathfrak{n} \geq 0}=\left\{f_{k, n}\right\}_{\mathfrak{n} \geq 0}$ and from equation (2), we have

$$
\mathbf{w}(\alpha)=\lim _{\mathfrak{n} \rightarrow \infty} s_{\mathfrak{n}}=\mathbf{f}_{\mathrm{k}} .
$$


Remark. Note that

$$
[0, \bar{k}]=\frac{1}{k+\frac{1}{k+\frac{1}{k+\frac{1}{\ddots}}}}=\frac{-k+\sqrt{k^{2}+4}}{2}=-r_{k, 2}
$$

From the above theorem, we conclude that k-Fibonacci words are Sturmian words.

A fractional power is a word of the form $z=x^{n} y$, where $n \in \mathbb{Z}^{+}$and $x \in \Sigma^{+}$, and $y$ is power power prefix of $x$. If $|z|=p$ and $|x|=q$, we say that $z$ is a $\mathrm{p} / \mathrm{q}$-power, or $z=x^{\mathrm{p} / \mathrm{q}}$. In the expression $x^{\mathrm{p} / \mathrm{q}}$, the number $\mathrm{p} / \mathrm{q}$ is the power's exponent. For example, 01201201 is a 8/3-power, $01201201=(012)^{8 / 3}$. The index of an infinite word $\mathbf{w} \in \Sigma^{\omega}$ is defined by

$$
\operatorname{Ind}(w):=\sup \{r \in \mathbb{Q} \geqslant 1: w \text { contains an } r \text {-power }\}
$$

For example $\operatorname{Ind}(\mathbf{f})>3$ because the cube $(010)^{3}$ occurs in $\mathbf{f}$ at position 6 . In $[15]$ the authors proof that $\operatorname{Ind}(\mathbf{f})=2+\phi \approx 3.618$. A general formula for the index of a Sturmian word was given in [8].

Theorem 18 If $\mathbf{u}$ is a Sturmian word of slope $\alpha=\left[0, a_{1}, a_{2}, a_{3}, \ldots\right]$, then

$$
\operatorname{Ind}(w)=\sup _{n \geqslant 0}\left\{2+a_{n+1}+\frac{q_{n-1}-2}{q_{n}}\right\},
$$

where $\mathrm{q}_{\mathrm{n}}$ is the denominator of $\alpha=\left[0, \mathrm{a}_{1}, \mathrm{a}_{2}, \mathrm{a}_{3}, \ldots, \mathrm{a}_{\mathrm{n}}\right]$ and satisfies $\mathrm{q}_{-1}=$ $0, q_{0}=1, q_{n+1}=a_{n+1} q_{n}+q_{n-1}$.

Corollary 19 The index of $\mathrm{k}$-Fibonacci words is $\operatorname{Ind}\left(\mathbf{f}_{\mathrm{k}}\right)=2+\mathrm{k}+\frac{1}{\mathrm{r}_{\mathrm{k}, 1}}$.

Proof. $\mathbf{f}_{\mathrm{k}}$ is a Sturmian word of slope $\alpha=[0, \bar{k}]$, then it is clear that $\mathrm{q}_{\mathrm{n}}=$ $\mathrm{F}_{k, n+1}$, and from above theorem

$$
\operatorname{Ind}\left(\mathbf{f}_{k}\right)=\sup _{n \geqslant 0}\left\{2+k+\frac{F_{k, n}-2}{F_{k, n+1}}\right\}=2+k+\frac{1}{r_{k, 1}} .
$$




\section{The k-Fibonacci Word Curve}

The Fibonacci word can be associated to a curve from a drawing rule. We must travel the word in a particular way, depending on the symbol read a particular action is produced, this idea is the same as that used in the L-Systems [17]. In this case, the drawing rule is called "odd-even drawing rule" [16], this is defined as shown in the following table.

\begin{tabular}{|c|l|}
\hline Symbol & Action \\
\hline 1 & Draw a line forward. \\
\hline 0 & $\begin{array}{l}\text { Draw a line forward and if the symbol } 0 \text { is in a position even } \\
\text { then turn } \theta \text { degree and if } 0 \text { is in a position odd then turn } \\
-\theta \text { degrees. }\end{array}$ \\
\hline
\end{tabular}

Definition 20 The $\mathrm{n}$ th-curve of Fibonacci, denoted by $\mathcal{F}_{\mathrm{n}}$, is obtained by applying the odd-even drawing rule to the word $\mathrm{f}_{\mathrm{n}}$. The Fibonacci word fractal $\mathcal{F}$, is defined as

$$
\mathcal{F}:=\lim _{n \rightarrow \infty} \mathcal{F}_{n} .
$$

Example 21 In Figure 1 we show the curve $\mathcal{F}_{10}$ and $\mathcal{F}_{17}$. The graphics in this paper were generated using the software Mathematica 9.0, [20].
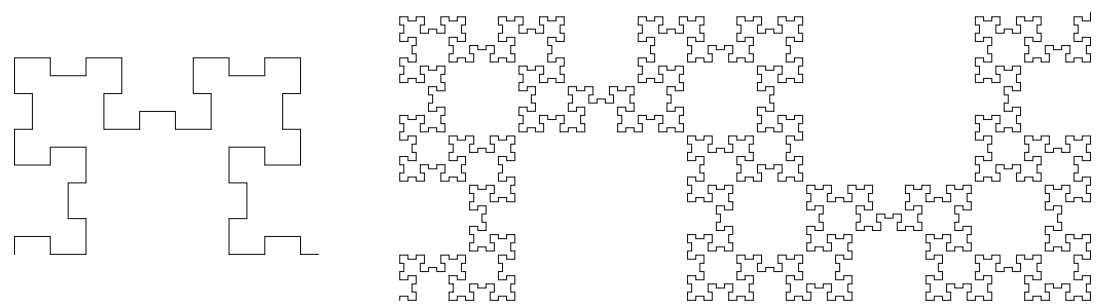

Figure 1: Fibonacci curves $\mathcal{F}_{10}$ and $\mathcal{F}_{17}$ corresponding to the words $f_{10}$ and $f_{17}$

Properties of Fibonacci Word Fractal can be found in $[3,4,16]$.

Definition 22 The $\mathrm{n}$ th $\mathrm{k}$-curve of Fibonacci, denoted by $\mathcal{F}_{\mathrm{k}, \mathrm{n}}$, is obtained by applying the odd-even drawing rule to the word $\mathrm{f}_{\mathrm{k}, \mathrm{n}}$. The $\mathrm{k}$-Fibonacci word curve $\mathcal{F}_{\mathrm{k}}$ is defined as

$$
\mathcal{F}_{k}:=\lim _{n \rightarrow \infty} \mathcal{F}_{k, n} .
$$




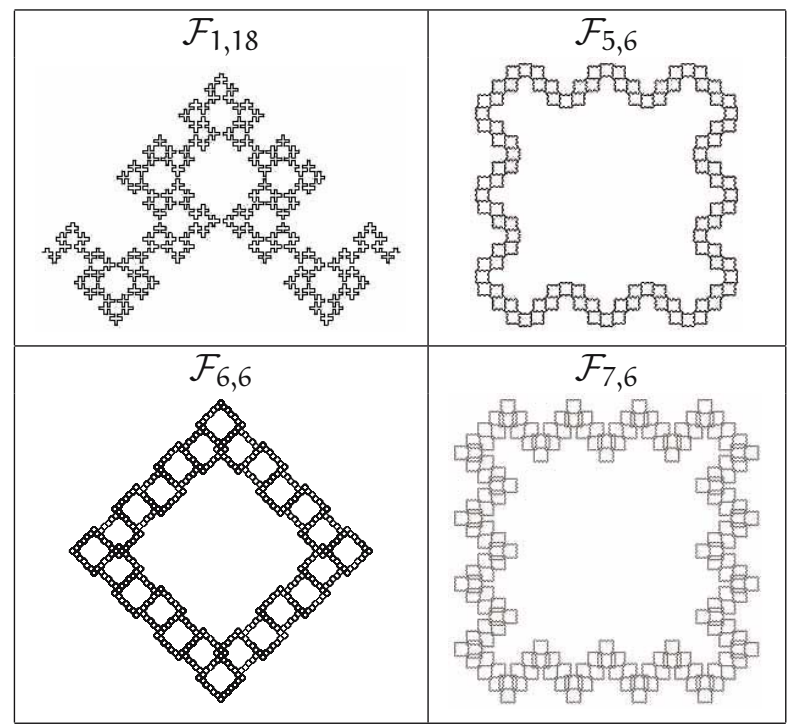

Table 1: Some curves $\mathcal{F}_{k, n}$ with $\theta=90^{\circ}$

In Table 1 , we show some curves $\mathcal{F}_{k, n}$ with an angle $\theta=90^{\circ}$.

In Table 2, we show some curves $\mathcal{F}_{k, n}$ with an angle $\theta=60^{\circ}$. In general these curves have a lot of patterns because the index is large, see Corollary 19.

Proposition 23 The k-Fibonacci word curve and the curve $\mathcal{F}_{\mathrm{k}, \mathrm{n}}$ have the following properties:

1. The k-Fibonacci curve $\mathcal{F}_{\mathrm{k}}$ is composed only of segments of length 1 or 2.

2. The $\mathcal{F}_{\mathrm{k}, \mathrm{n}}$ is symmetric.

3. The number of turns in the curve $\mathcal{F}_{\mathrm{k}, \mathrm{n}}$ is $\mathrm{F}_{\mathrm{k}, \mathrm{n}}+\mathrm{F}_{\mathrm{k}, \mathrm{n}-1}$.

4. If $\mathrm{n}$ is even then the $\mathcal{F}_{\mathrm{k}, \mathrm{n}}$ curve is similar to the curve $\mathcal{F}_{\mathrm{k}, \mathrm{n}-2}$ and if $\mathrm{n}$ is odd then the $\mathcal{F}_{\mathrm{k}, \mathrm{n}}$ curve is similar to the curve $\mathcal{F}_{\mathrm{k}, \mathrm{n}-3}$.

\section{Proof.}

1. It is clear from Proposition 12-1, because 110 and 111 are not subwords of $\mathbf{f}_{\mathrm{k}}$.

2. It is clear from Theorem 15 , because $f_{k, n}=\Phi\left(f_{k, n}\right) a b$, where $\Phi\left(f_{k, n}\right)$ is a palindrome.

3. It is clear from definition of odd-even drawn rule and because $\left|f_{k, n+1}\right|_{0}=$ $F_{k, n+1}+F_{k, n}$. 


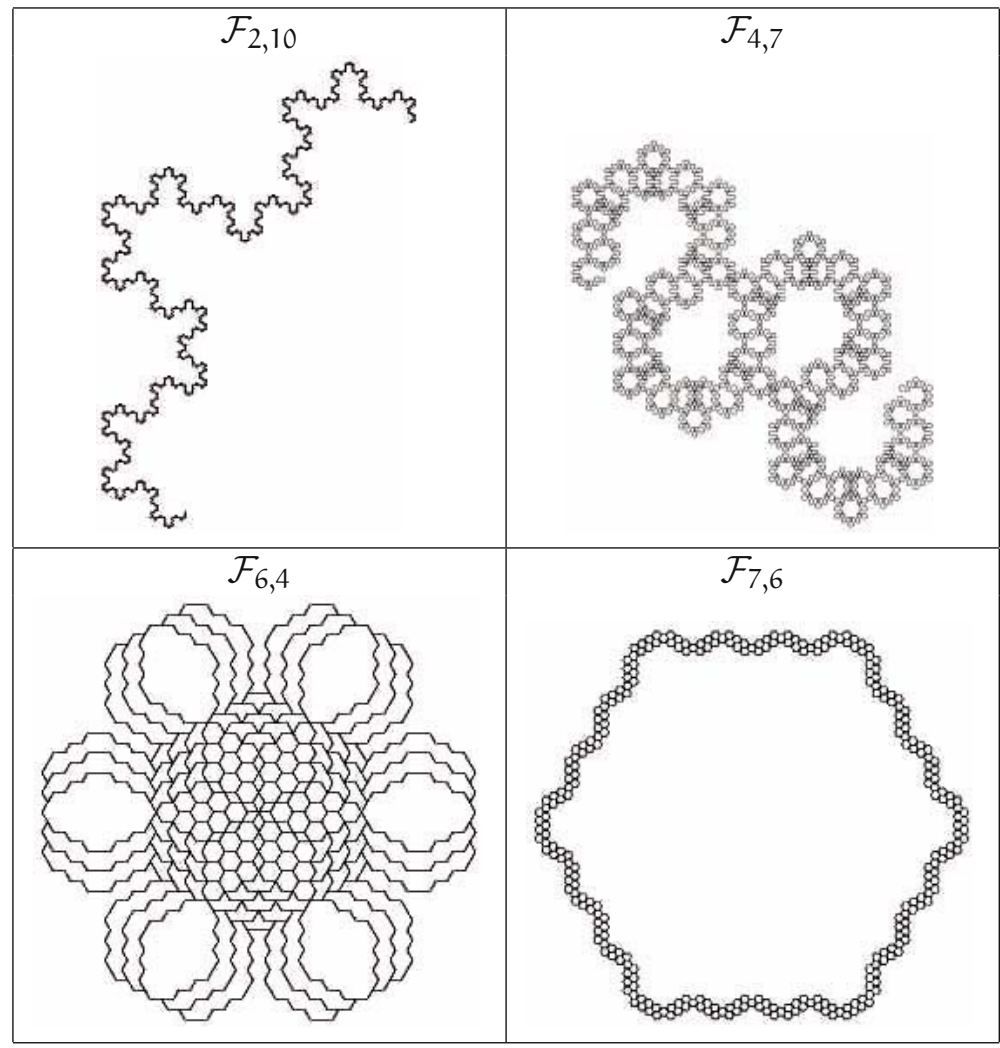

Table 2: Some curves $\mathcal{F}_{k, n}$ with $\theta=60^{\circ}$

4. If $n$ is even. It is clear that $\sigma_{k}^{2}\left(f_{k, n-2}\right)=f_{k, n}$. We are going to proof that $\sigma_{k}^{2}$ guaranties the odd-even alternation required by the odd-even drawing rule. In fact, $\sigma_{k}^{2}(0)=\sigma_{k}\left(0^{k-1} 1\right)=\left(0^{k-1} 1\right)^{k} 0$ and $\sigma_{k}^{2}(1)=\left(0^{k-1} 1\right)^{k} 0^{k} 1$. As $k$ is even, then $\left|\sigma_{k}^{2}(0)\right|$ and $\left|\sigma_{k}^{2}(1)\right|$ are odd. Hence if $|w|$ is even (odd) then $\left|\sigma_{k}^{2}(w)\right|$ is even (odd). Since $\sigma_{k}^{2}$ preserves the parity of length then any subword in the k-Fibonacci word preserves the parity of position.

Finally, we have to proof that the resulting angle of a pattern must be preserved or inverted by $\sigma_{k}^{2}$. Let $a(w)$ be the function that gives the resulting angle of a word $w$ through the odd-even drawing rule of angle 
$\theta$. Note that $a(00)=0^{\circ}, a(01)=-\theta^{\circ}$ and $a(10)=\theta^{\circ}$. Therefore

$$
\begin{aligned}
a\left(\sigma_{k}^{2}(00)\right) & =a\left(\left(0^{k-1} 1\right)^{k} 0\left(0^{k-1} 1\right)^{k} 0\right) \\
& =a\left(\left(0^{k-1} 1\right)^{k}\right) a\left(00^{k-1}\right) a\left(1\left(0^{k-1} 1\right)^{k-1} 0\right) \\
& =-k \theta^{\circ}+0^{\circ}+k \theta^{\circ}=0^{\circ} \\
a\left(\sigma_{k}^{2}(01)\right) & =a\left(\left(0^{k-1} 1\right)^{k} 0\left(0^{k-1} 1\right)^{k} 0^{k} 1\right) \\
& =a\left(\left(0^{k-1} 1\right)^{k}\right) a\left(00^{k-1}\right) a\left(1\left(0^{k-1} 1\right)^{k-1} 0\right) a\left(0^{k-1} 1\right) \\
& =-k \theta^{\circ}+0^{\circ}+k \theta^{\circ}-\theta^{\circ}=-\theta^{\circ} \\
a\left(\sigma_{k}^{2}(10)\right) & =a\left(\left(0^{k-1} 1\right)^{k} 0^{k} 1\left(0^{k-1} 1\right)^{k} 0\right) \\
& =a\left(\left(0^{k-1} 1\right)^{k}\right) a\left(0^{k}\right) a\left(1\left(0^{k-1} 1\right)^{k} 0\right) \\
& =-k \theta^{\circ}+0^{\circ}+(k+1) \theta^{\circ}=\theta^{\circ}
\end{aligned}
$$

Then $\sigma_{k}^{2}$ inverts the resulting angle, i.e., $a(w)=-a\left(\sigma_{k}^{2}(w)\right)$ for any word $w$. Therefore the image of a pattern by $\sigma_{k}^{2}$ is the rotation of this pattern by a rotation of $-\theta^{\circ}$. Since $\sigma_{k}^{2}\left(f_{k, n-2}\right)=f_{k, n}$, then the curve $\mathcal{F}_{k, n}$ is similar to the curve $\mathcal{F}_{k, n-2}$.

If $n$ is odd the proof is similar, but using $\sigma_{k}^{3}$.

Example 24 In Figure $2 \mathcal{F}_{4,4}$ looks similar to $\mathcal{F}_{4,6}, \mathcal{F}_{4,8}$ and so on.
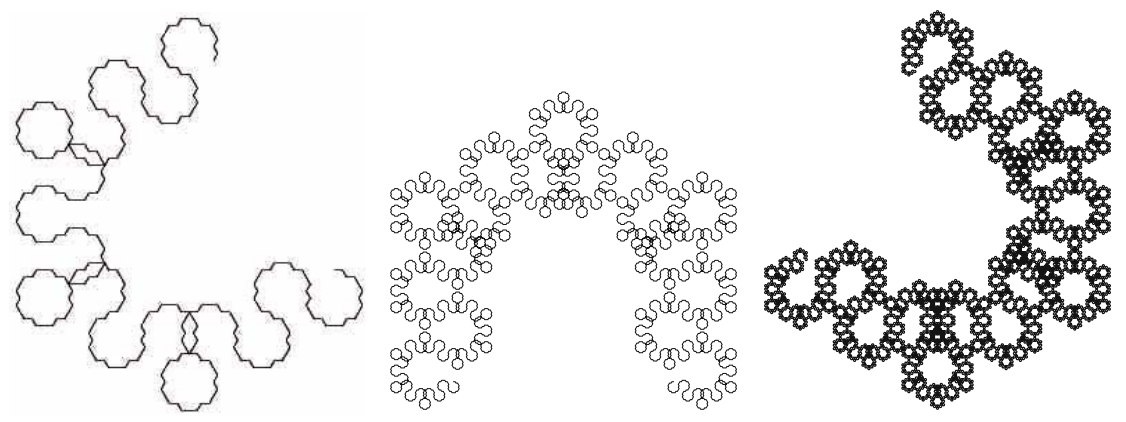

Figure 2: Curves $\mathcal{F}_{4,4}, \mathcal{F}_{4,6}, \mathcal{F}_{4,8}$ with $\theta=60^{\circ}$

In Figure $3 \mathcal{F}_{5,3}$ looks similar to $\mathcal{F}_{5,6}$.

\section{Acknowledgements}

The first author was partially supported by Universidad Sergio Arboleda under grant number USA-II-2012-14. 


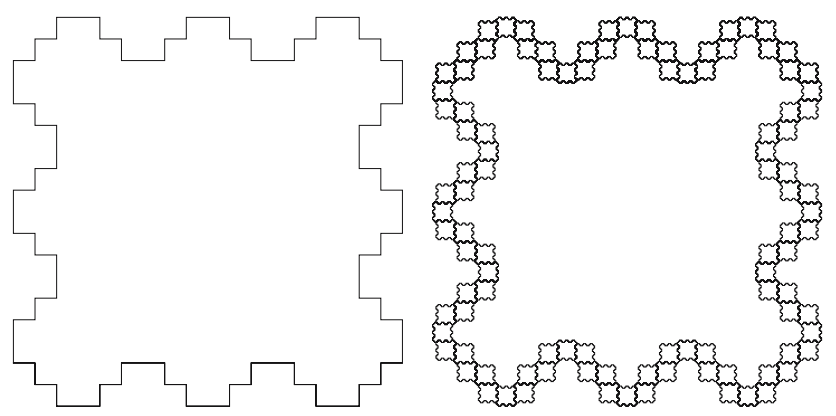

Figure 3: Curves $\mathcal{F}_{5,3}, \mathcal{F}_{5,6}$ with $\theta=60^{\circ}$

\section{References}

[1] J. Allouche, J. Shallit, Automatic Sequences, Cambridge University Press, 2003. $\Rightarrow 214,216$

[2] J. Berstel, Fibonacci words - a survey, in: G. Rosenberg, A. Salomaa (Eds.), The Book of L, Springer, Berlin, 1986, pp. 11-26. $\Rightarrow 213$

[3] A. Blondin-Mass, S. Brlek, A. Garon, S. Labb, Two infinite families of polyominoes that tile the plane by translation in two distinct ways, Theoret. Comput. Sci. 412 (2011) 4778-4786. $\Rightarrow 213,221$

[4] A. Blondin-Mass, S. Brlek, S. Labb, M. Mends France, Complexity of the Fibonacci snowflake, Fractals 20 (2012) 257-260. $\Rightarrow 221$

[5] C. Bolat, H. Kse, On the properties of k-Fibonacci numbers, Int. J. Contemp. Math. Sciences, 22, 5 (2010) 1097-1105. $\Rightarrow 213$

[6] J. Cassaigne, On extremal properties of the Fibonacci word, RAIRO - Theor. Inf. Appl. 42, (4) (2008) 701-715. $\Rightarrow 213$

[7] W. Chuan, Fibonacci words, Fibonacci Quart., 30, 1 (1992) 68-76. $\Rightarrow 213$

[8] D. Damanik, D. Lenz, The index of Sturmian sequences, European J. Combin., 23 (2002) 23-29. $\Rightarrow 220$

[9] A. de Luca, Sturmian words: structure, combinatorics, and their arithmetics, Theor. Comput. Sci. 183, 1 (1997) 45-82. $\Rightarrow 213$

[10] S. Falcon, A. Plaza, On k-Fibonacci sequences and polynomials and their derivatives, Chaos Solitons Fractals 39, 3 (2009) 1005-1019. $\Rightarrow 213$

[11] S. Falcon, A. Plaza, On the Fibonacci k-numbers, Chaos Solitons Fractals 32, 5 (2007) 1615-1624. $\Rightarrow 212,213$

[12] S. Falcon, A. Plaza, The k-Fibonacci sequence and the Pascal 2-triangle, Chaos Solitons Fractals 33, 1 (2007) 38-49. $\Rightarrow 213$

[13] T. Koshy, Fibonacci and Lucas numbers with Applications, Wiley-Interscience, 2001. $\Rightarrow 212$

[14] M. Lothaire, Algebraic Combinatorics on Words, Encyclopedia of Mathematics and its Applications, Cambridge University Press, 2002. $\Rightarrow$ 213, 214, 215, 216 
[15] F. Mignosi, G. Pirillo, Repetitions in the Fibonacci infinite word, RAIRO Theor. Inf. Appl. 26 (1992) 199-204. $\Rightarrow 213,220$

[16] A. Monnerot, The Fibonacci word fractal, preprint, 2009. $\Rightarrow 213,221$

[17] P. Prusinkiewicz, A. Lindenmayer, The Algorithmic Beauty of Plants, SpringerVerlag, Nueva York, 2004. $\Rightarrow 221$

[18] J. Ramírez, Incomplete k-Fibonacci and k-Lucas numbers, Chinese Journal of Mathematics (2013). $\Rightarrow 213$

[19] J. Ramírez. Some properties of convolved k-Fibonacci numbers. ISRN Combinatorics (2013) ID759641, 5pp. $\Rightarrow 213$

[20] J. Ramírez, G. Rubiano, Generating fractals curves from homomorphisms between languages [with Mathematica ${ }^{\circledR}$ ] (Spanish), Rev. Integr. Temas Mat. 30, 2 (2012) 129-150. $\Rightarrow 221$

[21] J. Ramírez, G. Rubiano, R. de Castro, A generalization of the Fibonacci word fractal and the Fibonacci snowflake, preprint arXiv:1212.1368, 2013. $\Rightarrow 213$

[22] W. Rytter, The structure of subword graphs and suffix trees of Fibonacci words, Theoret. Comput. Sci. 363, 2 (2006) 211-223. $\Rightarrow 213$

[23] A. Salas. About k-Fibonacci numbers and their associated numbers, Int. Math. Forum. 50, 6 (2011) 2473-2479. $\Rightarrow 213$

Received: June 12, 2013・Revised: October 25, 2013 